Journal for ImmunoTherapy of Cancer

\title{
Distinct mutational profile and immune microenvironment in microsatellite- unstable and POLE-mutated tumors
}

\author{
Hee Sang Hwang (D), Deokhoon Kim, Jene Choi
}

To cite: Hwang HS, Kim D, Choi J. Distinct mutational profile and immune microenvironment in microsatellite-unstable and POLE-mutated tumors. Journal for ImmunoTherapy of Cancer 2021;9:e002797. doi:10.1136/ jitc-2021-002797

- Additional supplemental material is published online only. To view, please visit the journal online (http://dx.doi.org/10. 1136/jitc-2021-002797).

Accepted 20 August 2021

Check for updates

(C) Author(s) (or their employer(s)) 2021. Re-use permitted under CC BY-NC. No commercial re-use. See rights and permissions. Published by BMJ.

Department of Pathology, Asan Medical Center, University of Ulsan College of Medicine, Seoul, South Korea

Correspondence to

Dr Jene Choi;

jenec@amc.seoul.kr

\section{ABSTRACT}

Introduction Mismatch repair (MMR)-deficient and DNA polymerase epsilon (POLE)-mutated tumors exhibit a high tumor mutation burden (TMB) and have been proven to be associated with good responses to immune checkpoint inhibitor treatments. However, the relationship between mutational characteristics of MMR-deficient and POLE-mutated tumors and the spatial architecture of tumor-infiltrating lymphocytes (TILs) has not been fully evaluated.

Methods We retrieved microsatellite instability-high (MSI-high, $\mathrm{N}=20$ ) and POLE-mutated ( $\mathrm{N}=47$ ) cases from the clinical next-generation sequencing cohort at Asan Medical Center. Whole-slide immunostaining for CD3, CD4, CD8, FoxP3 and PD-1 were performed with tissue samples of colorectal and gastric cancer $(\mathrm{N}=24)$ and the tumorpositive TIL cell densities were correlated with the tumor's mutational features. The findings were compared with the results of similar analyses in The Cancer Genome AtlasColorectal Adenocarcinoma (TCGA-COADREAD) cohort $(\mathrm{N}=592)$.

Results The MSI-high group showed significantly higher overall TMBs with a number of insertion/deletion (indel) mutations relative to the POLE-mutated group (median TMB; 83.6 vs 12.5/Mb). Oncogenic/likely-oncogenic POLE mutations were identified with ultrahypermutations ( $\geq 100$ mutations/Mb) (2/47, 4.3\%). Concurrent POLE mutations of unknown significance and MSI-high cases were identified in eight cases $(8 / 67,11 \%)$, and two of these colorectal cancers had multiple POLE mutations, showing an ultramutated phenotype (378.1 and 484.4/ $\mathrm{Mb}$ ) and low indel mutation burdens with complete loss of MSH-6 or PMS-2, which was similar to the mutational profile of the POLE-inactivated tumors. Intratumoral CD3-positive, CD4-positive, CD8-positive, FoxP3-positive and PD-1-positive TIL cell densities were more strongly correlated with the indel mutation burden than with the total TMB (correlation coefficient, $0.61-0.73$ vs $0.23-$ 0.38). In addition, PI3K/AKT/mTOR pathway mutations were commonly found in MSI-high tumors (75\%) but not in POLE-mutated tumors.

Conclusions Indel mutation burden rather than total TMB could serve as a predictor of high TILs in both MSI-high and POLE-mutated tumors. Multiple uncharacterized/ non-pathogenic POLE mutations occurring via MMR deficiency within MSI-high tumors may have combined pathogenic roles. A mutated PI3K/AKT/mTOR pathway may be a biomarker that can be used to stratify patients with advanced MSI-high tumors for immune therapy.

\section{INTRODUCTION}

Targeting immune evasion of the tumor has become a promising antineoplastic treatment modality. ${ }^{1}$ Recent genomic studies suggested that a high tumor mutation burden (TMB) confers an increased immune reaction to tumors and a better response to immune checkpoint inhibitor (ICI) treatment. ${ }^{2}$ In line with these observations, microsatellite instability (MSI)-high solid tumors became the first FDA-approved subject for ICI treatment. ${ }^{3}$ Furthermore, solid malignant tumors with POLE or POLD1 inactivation-associated proofreading deficiency in another type of hypermutated malignancy are also considered to be a good target for ICI treatment. ${ }^{4}$ High TMB tumors with mutations not within the exonuclease domain of POLE/POLD1 showed a significant response to ICI therapy. ${ }^{5}$ However, recent research also suggested that not all tumors with a high TMB show high immune responses to tumors or favorable responses to ICI treatment. ${ }^{6} 7$ Therefore, additional biomarkers to more precisely predict each patient's response to ICI treatment, beyond the TMB, are required.

Tumor-infiltrating lymphocytes (TILs) are a parameter indicating the immune response to a tumor. ${ }^{8}$ Therefore, assessing the characteristics of TILs could stratify the patients with a favorable response to ICI treatment. In accordance with this notion, many studies have reported that the amount and composition of TILs are correlated with a better response to ICI treatment in various solid tumors. ${ }^{9-11}$ Studies based on the bioinformatics approach suggested TMB and inflammatory biomarkers (T cellinflamed gene expression profile and PD-L1 expression) could independently predict the immune response to ICI, ${ }^{12}$ but the relationship between the TMB and the immune microenvironment in MSI-high and POLEmutated tumors has not been fully evaluated yet. 
The goal of the present study was to evaluate the genetic characteristics of POLE-mutated and MSI-high tumors and their immune environment and to identify a subgroup of hypermutated tumors that respond better to ICI therapy.

\section{MATERIALS AND METHODS Patients}

This study was approved by the Institutional Review Board in Asan Medical Center with waiver of patient's informed consent (Approval No. 2018-1080). A total of 67 cases with MSI-high $(\mathrm{N}=20)$ and POLE mutations $(\mathrm{N}=47)$ were retrieved from the clinical targeted nextgeneration sequencing (NGS) cohort of Asan Medical Center (AMC OncoPanel v3S) from March 2017 to June 2018 (AMC cohort). Briefly, the genomic DNA of each case was extracted from the representative formalin-fixed paraffin-embedded (FFPE) tumor tissue. The estimated tumor purities were at least 35\% (range, 35\%-90\%). After library preparation, the samples were sequenced using the MiSeqDx sequencing system (Illumina, San Diego, California, USA). The FASTQ file of each case was aligned to the reference genome (GRCh37) using BWA V.0.5.9, ${ }^{13}$ Picard V.2.5.0 (http://broadinstitute. github.io/picard), and standard genome analysis toolkit (GATK) V.1.6.5 ${ }^{14}$ to generate an analysis-ready BAM file. The variants and copy number alterations were called from the BAM files using VarDict V.1.6 ${ }^{15}$ and CNVkit V.0.9.6, ${ }^{16}$ respectively. Finally, all identified variants were manually reviewed by the authors (HSH and DHK) under the IGV genomic viewer system. ${ }^{17}$ The list of oncogenic/ likely oncogenic $(\mathrm{ONC} / \mathrm{LO})$ mutations of particular genes was retrieved from the OncoKB database. ${ }^{18}$ MSIhigh cases were identified with the NGS-based MSI test according to a previously described method ${ }^{19}$ or from a PCR-based MSI test with/without expression loss of one or more of the four mismatch repair (MMR) proteins (MLH-1, MSH-2, MSH-6 and PMS-2) based on the revised Bethesda guidelines. ${ }^{20}$ The immunostaining slides of the MMR proteins were reviewed by the pathologist (HSH). The mutation profiles were visualized using $\mathrm{R}$ package maftools V.2.4.05. ${ }^{21}$

\section{Pathologic review and immunohistochemical staining}

Immunohistochemistry (IHC) staining of the MMR proteins (MLH-1, MSH-2, MSH-6 and PMS-2) was carried out for the cases with available residual FFPE tumor tissue and they were reviewed by the pathologist $(\mathrm{HSH})$. In addition, whole-slide IHC staining for CD3, CD4, CD8, FoxP3 and PD-1 was performed on tissues from surgically resected colorectal and gastric cancer $(\mathrm{GC})$ cases $(\mathrm{N}=24)$. Briefly, the $4 \mu \mathrm{m}$ thick representative sections of FFPE tumor tissues of each case were deparaffinized in xylene and hydrated in an ethanol series. After blocking endogenous peroxidases and heat-induced antigen retrieval, the tumor sections were incubated at room temperature for $32 \mathrm{~min}$. After washing, the tumor sections were further incubated with antimouse or antirabbit secondary antibodies for $30 \mathrm{~min}$. The sections were processed with an iView DAB detection kit (Ventana Medical Systems, Tucson, Arizona, USA) and counterstained with hematoxylin. The above procedures were carried out using the BenchMark XT autostainer system (Ventana). The manufacturers and dilution rates of the primary antibodies are described in online supplemental table 1.

\section{Digital image-based quantitation of the tumor immune cell population}

Whole slide images were obtained from the CD3, CD4, CD8, FoxP3 and PD-1 immunostaining slides using Panoramic 250 (3DHISTECH, Budapest, Hungary) to quantify the densities of the populations of TILs. The whole tumor area and outer invasive margin of each slide image was labeled using QuPath V.0.1.2. ${ }^{22}$ The outer invasive margin was defined to be a stromal area $500 \mu \mathrm{m}$ outside the tumor-stromal interface. The positive cells in the tumor areas were counted using Positive Cell Detection module in the QuPath program. The DAB signal cut-off of each of the immunostaining markers was repeatedly calibrated by the author ( $\mathrm{HSH})$. The positive cell densities of the tumor area and outer invasive margin were calculated as the number of positive cells divided by the tumor area measurements (cell counts $/ \mathrm{mm}^{2}$ ) (online supplemental figure 1A-C). We found that the immune cell densities of tumor area and outer invasive margin were strongly correlated $(\mathrm{R}=0.72-0.93, \mathrm{p}<0.001$ for all comparisons) (data not shown); therefore, we used the immune cell densities of the tumor area in additional analyses.

\section{Analysis of The Cancer Genome Atlas (TCGA) database}

The gene expression profile data of RNA-sequencing $(\mathrm{N}=592)$ and mutation annotation files $(\mathrm{N}=528)$ from the TCGA-Colorectal Adenocarcinoma (TCGA-COADREAD) cohort $(\mathrm{N}=592)$ were obtained. The resulting files of the TCGA-COADREAD dataset were downloaded from the cBioPortal cancer genomics database (https://www.cbioportal.org). ${ }^{23} 24$ The results of the mononucleotide and dinucleotide marker panel of the TCGA-COADREAD dataset were retrieved from the Broad GDAC firehose website (https://gdac.broadinstitute.org). Ninety-nine cases with MSI-high and/or POLE-mutated cases were further retrieved from the TCGA-COADREAD dataset and analyzed in a similar fashion as the AMC cohort. The signatures regarding tumor immunity, including lymphocyte infiltration and CD8 + T cell signatures, were retrieved from the supplementary data of the pan-immune feature matrix for the entire TCGA cohort analyzed by Thorsson et $a l^{25}$

\section{Statistical analysis}

Differences of continuous numerical variables between the groups were compared using the Mann-Whitney U test. The correlation between the two continuous numerical variables was evaluated using Pearson's correlation test. 
Table 1 Summary of the clinical features of the enrolled cases $(\mathrm{N}=67)$

\begin{tabular}{|c|c|c|c|c|}
\hline Parameters & & *MSI-high (N=20) (\%) & POLE-mutated (N=47) (\%) & Total (\%) \\
\hline Age (mean $\pm S D)$ & & $48.8 \pm 11.9$ & $61.4 \pm 11.7$ & $57.7 \pm 13.1$ \\
\hline Sex & Male & $14(70)$ & $30(64)$ & $44(66)$ \\
\hline \multirow[t]{7}{*}{ Type of malignancies } & CRC & $14(70)$ & $25(53)$ & $39(58)$ \\
\hline & NSCLC & 0 & $12(26)$ & $12(18)$ \\
\hline & GBM & $2(10)$ & 0 & $2(3)$ \\
\hline & OV & $1(5)$ & $1(2)$ & $2(3)$ \\
\hline & $\mathrm{BC}$ & 0 & $1(2)$ & $1(1.5)$ \\
\hline & SCLC & 0 & $1(2)$ & $1(1.5)$ \\
\hline & HNSCC & 0 & $1(2)$ & $1(1.5)$ \\
\hline
\end{tabular}

*Defined by the published criteria of Kim et al. ${ }^{19}$

BC, breast cancer; CRC, colorectal cancer; GBM, glioblastoma; GC, gastric cancer; HNSCC, head and neck squamous cell carcinoma; MSI, microsatellite instability; NSCLC, non-small cell carcinoma; OV, ovarian cancer; SCLC, small cell lung carcinoma.

The statistical analyses were carried out using R V.4.0.2 (R Foundation for Statistical Computing, Vienna, Austria). A two-sided $\mathrm{p}<0.05$ was considered to be significant.

\section{RESULTS}

\section{Clinicopathologic features of the patient cohorts}

A total of 67 patients were analyzed and their clinical features are shown in table 1 . The mean age of the patients in the MSI-high group was significantly lower than that in the POLE-mutated groups $(\mathrm{p}=0.0001)$. There was a male predominance in both the MSI-high and POLE-mutated groups. Colorectal cancer (CRC) was the most common cancer type in both the MSI-high and POLE-mutated groups, accounting for $70 \%$ of MSI-high and $53 \%$ of the POLE-mutated groups, respectively. Other types of malignancies included in the study were non-small cell lung cancer (NSCLC), GC, glioblastoma (GBM), ovarian cancer (OV), small cell lung cancer (SCLC), head and neck squamous cell carcinoma (HNSCC) and breast cancer (BC). MSI-high was not observed in patients with NSCLC, SCLC, HNSCC or BC. The NGS-based MSIhigh result was significantly correlated with the MSIhigh results from the clinically defined MMR phenotype evaluated by a PCR-based MSI test and/or MMR protein immunostaining $(\mathrm{p}<0.0001)$ with $100 \%$ specificity $(95 \%$
CI 0.85 to 1.00 ) and $79 \%$ sensitivity (95\% CI 0.54 to 0.94 ) (table 2).

\section{Mutational profile comparison of the MSI-high and POLE- mutated groups}

We next analyzed the mutational profile of the AMC cases (figure 1) with a focus on frequently mutated genes in MSIhigh tumors and oncogenic driver alterations in signaling pathways. The most common alterations in the MSI-high group were frameshift deletion mutations. Among them, CDH26 (70\%), ACVR2A (65\%) and RNF43 (50\%) were the most frequently mutated genes with homopolymer sequences in their coding regions. Interestingly, PIK $3 C A$ missense mutations were detected in $65 \%$ of the cases $(13 / 20)$, which was ONC/LO in 11 cases. Among the PIK3CA mutated tumors, colon cancer cases were predominant $(7 / 13,53 \%)$ (figure $1 \mathrm{~A})$. In contrast, the POLEmutated group exhibited various alterations, including mutations of common tumor suppressor genes such as TP53 (83\%) and APC (49\%), and subsequently KRAS (34\%) and FBXW7 (21\%), especially in CRCs (figure 1B). PIK3CA mutations were observed, but their frequency was much lower in the POLE-mutated group than the MSIhigh group (19\% vs 65\%). In terms of TMB, the MSI-high group showed significantly higher overall TMBs with a greater number of insertion/deletion (indel) mutations

Table 2 Comparison of next-generation sequencing-defined MSI-high group with the clinical MMR phenotype

\begin{tabular}{|c|c|c|c|c|}
\hline & & \multicolumn{2}{|l|}{ Clinical phenotype } & \multirow[b]{2}{*}{ Total (\%) } \\
\hline & & MMR-deficient (\%) & MMR-proficient (\%) & \\
\hline NGS phenotype & MSI-high & $15(79)$ & 0 & $15(36)$ \\
\hline Total & & $19(100)$ & $23(100)$ & $42(100)$ \\
\hline
\end{tabular}

Fisher's exact test $\mathrm{p}<0.0001$.

MMR, mismatch repair; MSI, microsatellite instability; MSS, microsatellite stable; NGS, next-generation sequencing. 

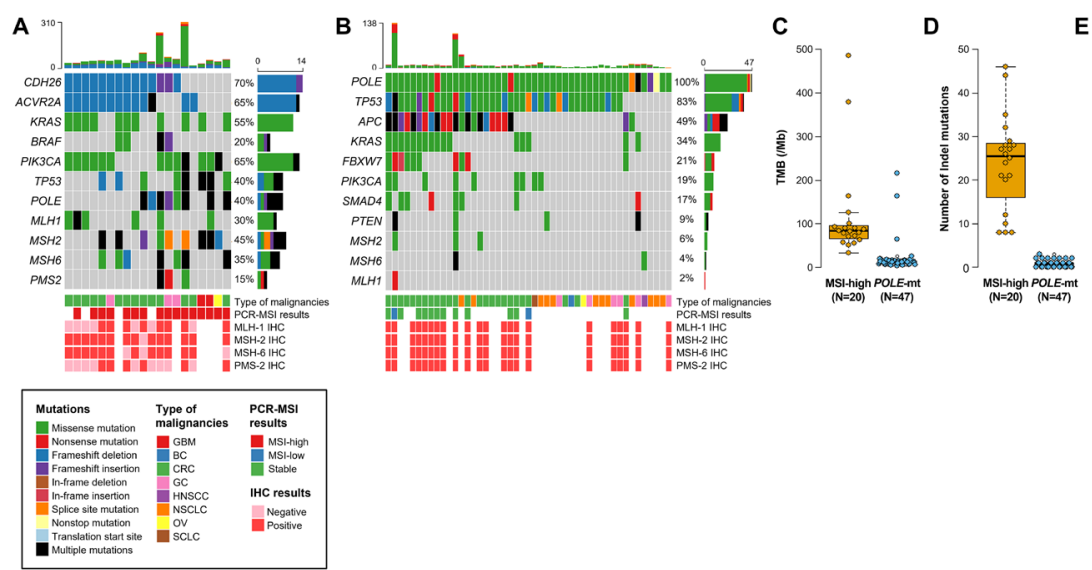

E

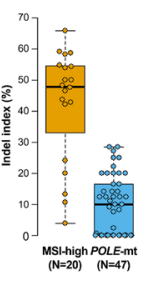

Figure 1 Mutation profiles with overall and indel mutation frequencies of MSI-high and POLE-mutant cases. (A-E) MSI-high and POLE-mutant cases of the AMC cohort. (A) Mutation profile of MSI-high cases $(\mathrm{N}=20)$. Note the high proportion of $A C V R 2 A$ frameshift deletion and PIK3CA mutations (65\% each). (B) Mutation profile of POLE-mutant cases ( $\mathrm{N}=47)$. Only a few cases $(\mathrm{N}=2)$ showed an ultrahypermutation ( $>100$ mutations/Mb) phenotype. (C-E) TMB (C), number of indel mutations (D) and indel index (indel mutation frequencies) (E), were significantly elevated in MSI-high cases ( $p<0.001$ for all comparisons). White-colored items indicates the data were not available. BC, breast cancer; CRC, colorectal cancer; GBM, glioblastoma; GC, gastric cancer; HNSCC, head and neck squamous cell carcinoma; MSI, microsatellite instability; NSCLC, non-small cell carcinoma; ONC/LO, oncogenic/likely oncogenic; OV, ovarian cancer; SCLC, small cell lung carcinoma; TMB, tumor mutation burden.

than the POLE-mutated group (median TMB; 83.6 vs $12.5 / \mathrm{Mb}$ ) (figure 1C-E). These results suggest that POLEmutated tumors have variable TMBs that depend on their specific POLE variants.

\section{MSI-high tumors show frequent genomic alterations in the PI3K/AKT/mTOR pathway}

We further investigated the mutational profile of canonical pathways, such as RTK/RAS/MAP-Kinase, TGFß/ SMAD, PIK3/AKT/mTOR and $\beta$-catenin/Wnt. As shown in figure 2 , we found that a substantial number of genes were mutated in the PI3K/AKT/mTOR pathway in the MSI-high group, including PI3K catalytic subunit genes (PIK3CB and PIK3CD), PI3K regulatory subunit genes (PIK3R1 and PIK3R2), AKT/protein kinase $\mathrm{B}$ genes (AKT1, AKT2 and AKT3), RICTOR, MTOR and PTEN, affecting 17 of 20 cases $(85 \%)$. In combination with the oncogenic mutations of the PI3KCA gene (65\%), overall 15 of 20 cases $(75 \%)$ displayed ONC/LO mutations involving the PI3K/AKT/mTOR pathway, suggesting that any genetic alterations in the PI3K/AKT/mTOR pathwayassociated genes play a significant role in the pathogenesis of MSI-high tumors. We did not identify any pattern of recurrent and functionally relevant mutations in the other pathways. Of note, the alterations of the PI3K/ AKT $/ \mathrm{mTOR}$ genes were not significantly associated with the increased immune cell density (data not shown).

\section{Not all POLE-mutated tumors are associated with a hypermutational phenotype or increased T-cell infiltration}

To correlate the association of different POLE mutations and their TMB in this cohort, we compared specific POLE

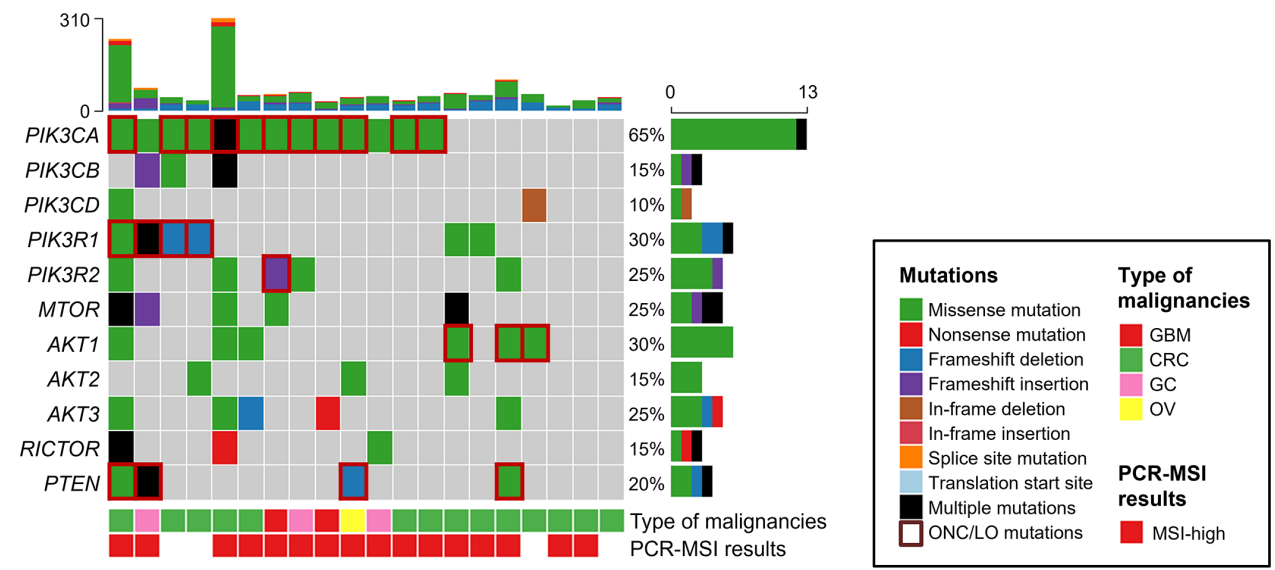

Figure 2 Mutation profile for the genes of the constituents of PI3K/Akt/mTOR pathways in MSI-high cases ( $\mathrm{N}=20)$. Known ONC/LO mutations involving the components of thePI3K/AKT/mTOR pathway were identified in 15 of 20 (75\%) of the MSIhigh cases. Among the MSI-high cases, 71\% were CRCs (10/14). ONC/LO mutations are labeled with red empty boxes. CRC, colorectal cancer; GC, gastric cancer; GBM, glioblastoma; MSI, microsatellite instability; ONC/LO, oncogenic/likely oncogenic; $\mathrm{OV}$, ovarian cancer. 


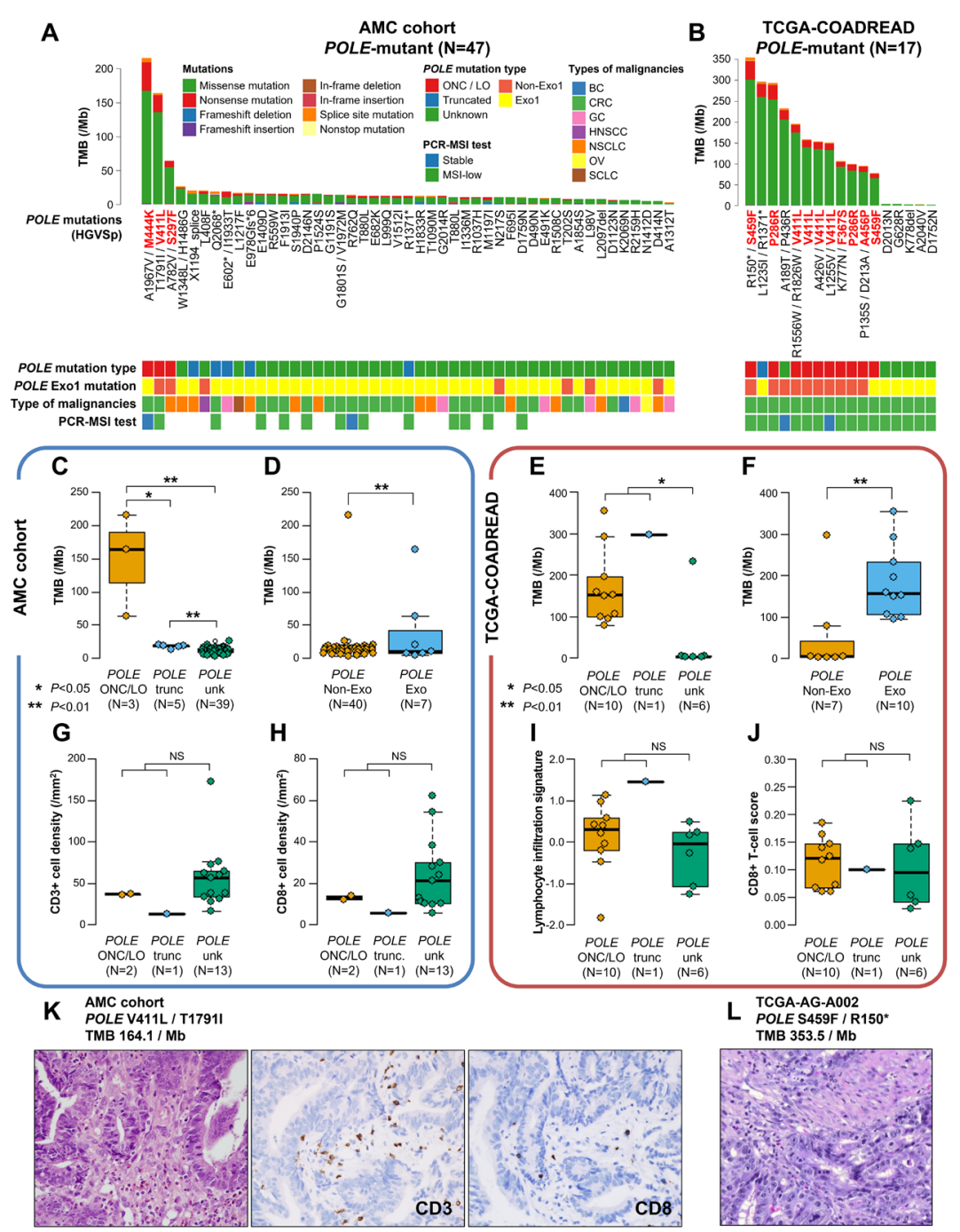

Figure $3 \mathrm{TMB}$ and T-cell infiltration densities of POLE-mutant cases regarding the characteristics of POLE mutations. (A) TMB of each POLE-mutant tumor in the AMC cohort. Predicted protein alterations of each case are presented. (B) TMB of each POLE-mutant tumor in the TCGA-COADREAD cohort. The cases harboring POLE ONC/LO mutations show a hypermutated phenotype. (C-F) TMB of POLE-mutant cases in the AMC and TCGA-COADREAD cohorts. (C) A few cases with POLE ONC/ LO mutations show a hypermutation phenotype. (D) The exonuclease domain POLE-mutated cases show higher TMBs than the outside-exonuclease domain POLE mutated cases, but the difference is not remarkable. (E) POLE ONC/LO mutations display a significantly high mutation load. (F) Cases with the POLE exonuclease domain exhibit remarkably high TMB, but these cases largely belong to the POLE ONC/LO mutation group. (G-J) Immune cell densities and immune cell signatures of POLE-mutant cases in AMC and TCGA-COADREAD cohorts, respectively. (G-H) The tumor CD3-positive (G) and CD8-positive (H) T-cell densities are not significantly different between the groups with POLE ONC/LO and truncated mutation and POLE unknown significance mutation. (I-J) Lymphocyte infiltration signature (I) and CD8 + T cell scores (J) of the POLE ONC/LO and truncated mutation groups are slightly elevated compared with the POLE unknown significance mutation group, but these findings do not reach statistical significance. (K) The representative histology of a POLE ONC/LO mutation case in the AMC cohort, showing a scanty number of CD3-positive and CD8-positive lymphocytes. (L) A case with high-TMB and double POLE ONC/ LO mutations from the TCGA-COADREAD cohort display a scanty amount of TILs. AMC, Asan Medical Center; BC, breast cancer; CRC, colorectal cancer; GBM, glioblastoma; GC, gastric cancer; HNSCC, head and neck squamous cell carcinoma; MSI, microsatellite instability; NSCLC, non-small cell carcinoma; ONC/LO, oncogenic/likely oncogenic; NS, non-significant; OV, ovarian cancer; SCLC, small cell lung carcinoma; TIL, tumor-infiltrating lymphocyte; TMB, tumor mutation burden; unk, unknown-significant.

mutations with their mutational profiles. As shown in the plots of the mutational landscape (figure 3A), a large number of POLE-mutated tumors did not show extraordinarily high TMBs. Only two POLE-mutated tumors exhibited an ultrahypermutated genotype ( $\geq 100$ mutations/ $\mathrm{Mb})$, which had ONC/LO POLE M444K and V411L mutations, and were both CRCs. In contrast, a NSCLC case with the ONC/LO POLE S297F mutation, which is one of the most common pathogenic POLE mutations, did not show an ultrahypermutation (TMB 64.0/Mb). In the comparisons using the mutational type, high TMB was frequently detected in tumors with ONC/LO POLE 
mutations with a median TMB of $164.1 \mathrm{mut} / \mathrm{Mb}$ followed by tumors with truncated (median TMB 18.8 mut/Mb) and unknown-significant (median TMB 10.9 mut/Mb) POLE mutations $(\mathrm{p}<0.05)$, and any unknown-significant POLE mutations, including truncated or the functional exonuclease domain mutations, did not show ultrahypermutation (figure 3C). Moreover, a TMB difference was not identified between the exonuclease domain and the outside-exonuclease domain POLE-mutated tumors (figure 3D). To further confirm our data, we analyzed the POLE-mutated cases from the TCGA-COADREAD cohort $(\mathrm{N}=17)$ and found that $\mathrm{ONC} / \mathrm{LO}$ POLE mutated tumors also showed also ultrahypermutation or hypermutation, while unknown-significant POLE mutated ones did not display hypermutation except for two cases (figure 3B,E), which are consistent with our results. Mutations within the POLE exonuclease domain are strongly associated with high TMB in the TCGA-COADREAD cohort, but this is attributable to the cases with ONC/LO POLE mutations, which are primarily located within the exonuclease domain (figure 3F).

We next evaluated the association of POLE mutations with the degree of tumorous immune cell infiltration. The two cases with ONC/LO POLE mutations in our AMC cohort (M444K and V411L) showed similar levels of CD3-positive and CD8-positive T cell densities compared with truncated or unknown-significant POLE-mutated tumors at the center of the resected gastric and CRCs (figure 3G,H). In the TCGA-COADREAD cohort, the scores of the lymphocyte infiltration signature and CD8 + Tcell were slightly elevated in the POLE ONC/ LO and truncated mutation groups, mostly being ultrahypermutated, compared with those with the unknown significant POLE mutated group, but the difference was not significant (figure 3I-J). Histologic images of ultrahypermutated resected CRCs with oncogenic POLE V411L (figure 3K) and $\mathrm{S} 459 \mathrm{~F}$ (figure 3L) mutations from our cohort and the TCGA-COADREAD cohort, respectively, showed scanty amounts of TILs.

Taken together, these data indicate that the large number of POLE mutations could be non-functional passenger mutations, and the unknown significant POLE mutations are generally not inducing hypermutation, unlike the ONC/LO POLE mutation. In immune responses, ONC/LO POLE mutated tumors with the ultrahypermutated genotypes did not directly lead to high immune responses in both cohorts.

\section{Tumors with concurrent MSI-high and POLE mutations}

We identified eight cases with concurrent MSI-high and POLE mutations (figure 4A) in GBM, CRC and GC in the AMC cohort. Among these cases, two CRC cases showed exceptionally high TMBs (Case 1, 484.4 mut/ $\mathrm{Mb}$; Case 2, $378.1 \mathrm{mut} / \mathrm{Mb})$, which is higher than that
A

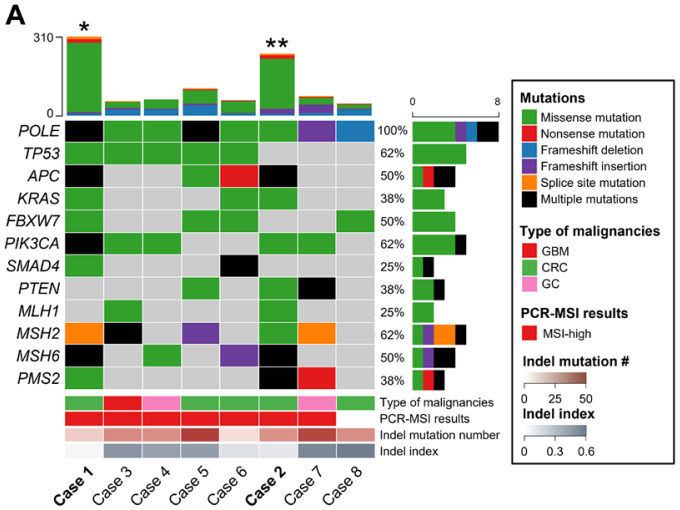

C Case 1: MSH6 $11239 \mathrm{Kfs} \times 35$, MSH2 $\times 820 \_$splice

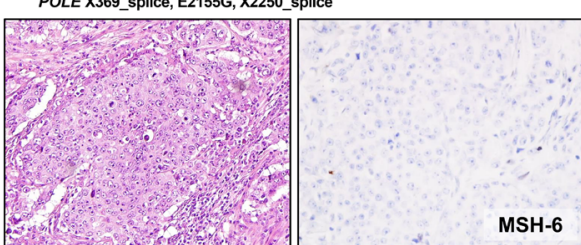

B

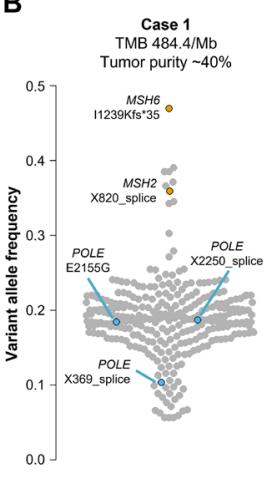

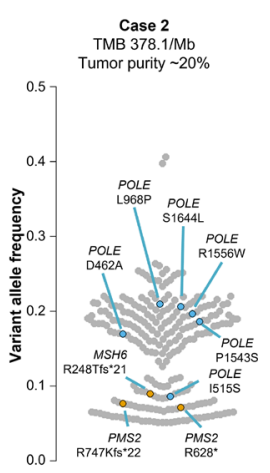

Figure 4 Characteristics of concurrent MSI-high and POLE-mutant cases. (A) Mutation profile of the cases with concurrent MSI-high and POLE mutations ( $\mathrm{N}=8$ ). Two of eight cases (labeled asterisks) exhibited exceptionally high TMBs (Case 1, 484.4/ $\mathrm{Mb}$; Case 2, 378.1/Mb), which are predominantly missense mutations. (B) Scatterplots of variant allele fractions of two cases with exceptionally high TMBs. MSH6 and MSH2 mutations appear to precede multiple POLE mutations in Case 1. In Case 2, variant allele frequencies of the POLE mutations were larger than those of the MSH6 and PMS2 gene mutations. However, the sum of the variant allele frequencies of the truncated PMS2 gene mutations was similar to the estimated tumor purity; hence, the PMS2 gene truncations appear to be a clonal event. (C) Histology of Case 1 and Case 2 showing poorly differentiated sheets of tumor cells with complete losses of MSH-6 and PMS-2 expression, respectively. CRC, colorectal cancer; GC, gastric cancer; GBM, glioblastoma; MSI, microsatellite instability; TMB, tumor mutation burden. 
of the MSI-high-only CRCs (median TMB, 83.6 mut/ $\mathrm{Mb}$ ), with distinct characteristics of a high missense mutation load (273 and 194 mutations) and a low indel index (3.9\% and $10.7 \%$ ), which is similar to that of the POLE inactivation-associated ultrahypermutation phenotype. These two cases were MSI-high tumors as confirmed by the PCR-based tests. Scatterplots of the variant allele fractions (VAFs) of Case 1 showed that VAFs of the MMR gene mutations were much higher than that of the POLE mutations after considering the approximate $40 \%$ tumor purity, suggesting that the MMR gene mutations may have preceded the POLE mutations during the development of the tumor (figure 4B). In Case 2, even though the VAFs of the multiple POLE mutations were higher than those of the MMR gene mutations, the sum of the VAFs of the two PMS2 mutations (R747Kfs*22 and R628*) was comparable to those of the POLE mutations, considering the estimated tumor purity of $20 \%$ (figure $4 \mathrm{~B}$ ), with a complete loss of PMS-2 expression (figure 4C). Thus, it appears that multiple POLE and PMS2 mutations were present in all of the cancer cells in Case 2. Except for the POLE X369_splice mutation, any of the concurrent POLE mutations in the first and second cases were not located within the POLE exonuclease domain (codon 86-426). The other six MSI-high cases showed single or multiple POLE mutations of unknown biological significance, which were probably subclonal because of their low VAFs (online supplemental figure 2). These results suggested that concurrent multiple POLE mutations, that may be either clonal or subclonal, may occur within MSI-high tumors. Some of these POLE mutations may be biologically pathogenic and result in an ultrahypermutation phenotype.

\section{MSI-high phenotype and indel mutation number, rather than POLE status or tumor mutation burden, are correlated with the tumor immune cell density}

We further evaluated the significance of the MSI-high, POLE-mutated phenotypes and the TMB on TILs with colorectal and GC (N=24) from our cohort. MSI-high tumors showed significantly higher CD4-positive and CD8positive T-cell infiltrations compared with microsatellitestable (MSS) tumors. CD3-positive, FoxP3-positive and PD-1-positive immune cell densities were also increased in the MSI-high tumors, although the increase did not reach statistical significance (figure $5 \mathrm{~A}-\mathrm{G}$ ). Unexpectedly, ONC/LO POLE-mutated tumors with ultrahypermutation did not show significant differences in terms of immune cell infiltration compared with unknownsignificant POLE-mutated tumors (figure 5A-G). Similarly, the mRNA expression levels of T-cell markers (CD3D, $C D 4$ and $C D 8 A$ ) and lymphocyte infiltration signature scores were slightly elevated in MSI-high tumors, but the differences were not statistically significant except for CD8A expression (online supplemental figure $3 \mathrm{~A}-\mathrm{D}$ ) in the TCGA-COADREAD cohort.

In the analyses of the relationship between the immune cell infiltration, TMBs and indel mutations, the number of indel mutations was strongly correlated with all examined types of immune cell (CD3+, CD4+, CD8+, FoxP3 + and $\mathrm{PD}-1+$ ) densities $(\mathrm{R}=0.61-0.72, \mathrm{p} \leq 0.002)$ (figure $5 \mathrm{H}-\mathrm{L}$ ), whereas the total number TMB was not significantly associated with all examined immune cell densities $(\mathrm{R}=0.23-$ 0.38); only CD3-positive and CD4-positive T-cell densities showed borderline significance ( $\mathrm{p}=0.072$ for both cases) (figure 5M-Q). These data demonstrated that a high indel mutation load might be a more useful predictor of tumor immunogenicity than total TMB in MSI-high and POLE-mutated tumors. In the same analyses with the TCGA-COADREAD cohort, neither indel mutation number nor TMB were associated with T-cell marker expressions or lymphocyte infiltration signature, but the expression of $C D 3 D$ and indel mutation number showed a marginal association (online supplemental figure $3 \mathrm{E}-\mathrm{L}$ ). The observed differences in our AMC cohort and the TCGA-COADREAD cohort may be due to variable factors, including tumor cell content and different evaluation methods (IHC vs mRNA expression).

\section{DISCUSSION}

In this study, we found that MSI-high and POLE-mutated tumors exhibited different mutation profiles. MSI-high tumors exhibited frequent $\mathrm{PI} 3 \mathrm{~K} / \mathrm{AKT} / \mathrm{mTOR}$ pathway alterations with high TMBs (median TMB; 83.6), but POLE-mutated tumors displayed heterogeneous genetic alterations and a variable TMB (median TMB; 12.5/ $\mathrm{Mb})$. Of note, only ONC/LO POLE mutations were almost exclusively associated with the ultrahypermutation genotype, but some of the cases with unknownsignificant POLE mutations with or without a concurrent MSI-high phenotype showed high TMBs similar to those of the ONC/LO POLE inactivated cases. Rather than the TMB, the number of indel mutations was more strongly correlated with the densities of the TILs. These results suggest the importance of indel mutations rather than the total number of mutation events for predicting a clinical benefit from immunotherapy.

One of the ongoing debates in immunotherapy is whether there is a tumor genomic determinant associated with the immune fitness of a specific tumor that can be used to stratify patients for immune therapy. In some studies, high non-synonymous TMB is a predictive biomarker for the response to monoclonal antibody therapy directed against the PD-1 receptor, namely pembrolizumab; ${ }^{26}$ meanwhile, other studies showed that the neoantigens produced by indel mutations could trigger an antineoplastic immune response. ${ }^{27} 28$ Consistent with previous studies depicting the positive relationship between the indel mutation load and tumorous immune response in MSI-high CRCs, ${ }^{29}{ }^{30}$ we also demonstrated that MSI-high tumors showed significantly high levels of intratumoral CD4-positive and CD8-positive T-cell densities compared with MSS tumors. In addition, an important point that should be emphasized in our data is that the indel mutation load was significantly correlated with the infiltration 

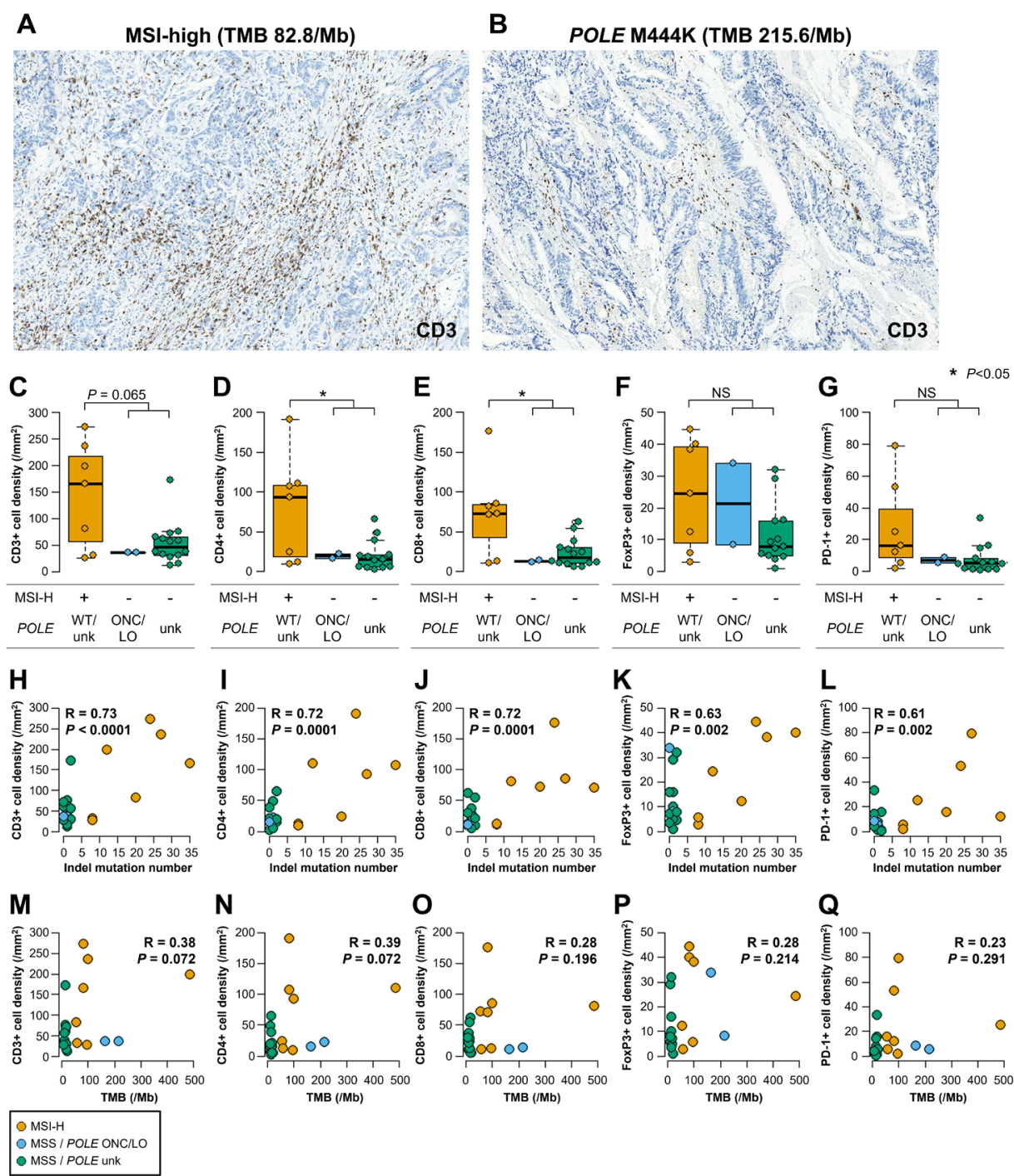

Figure 5 Densities of immune cell infiltrations in MSI-high and POLE-mutant cases in the AMC cohort. (A,B) Photomicrography of CD3 immunostaining with POLE M444K (TMB: 215.6/Mb) and MSI-high (TMB: 82.8/Mb) cases. The amount of CD3-positive T-cell infiltration is lower in the POLE M444K case even though it is high TMB. (C-G) The amount of immune cells positive for CD3 (C), CD4 (D), CD8 (E), FoxP3 (F) and PD-1 (G) are compared according to the MSI and POLE mutation statuses. CD4-positive and CD8-positive cell densities were significantly increased in the MSI-high group $(p<0.05)$. CD3-positive cell densities were also elevated in the MSI-high group, but this was not statistically significant $(p=0.065)$. $(\mathrm{H}-\mathrm{L})$ The number of indel mutations were significantly correlated with CD3-positive (C), CD4-positive (D), CD8-positive (E), FoxP3-positive (F) and PD-1-positive (G) immune cell infiltration densities in all MSI-high and POLE-mutant cases. (M-Q) In contrast, overall TMB was not significantly correlated with the tumorous immune cell infiltration densities. AMC, Asan Medical Center; MSI, microsatellite instability; ONC/LO, oncogenic/likely oncogenic; NS, non-significant; TMB, tumor mutation burden.

of the above immune cells in the tumors, while total TMB appears to not be as strongly associated with TIL densities. These findings were not completely consistent with those from the TCGA-COADREAD cohort. There are important caveats to using TCGA data due to the characteristics of bulky sequencing data that may not delicately quantify the amounts of TILs compared with the IHC staining-based methods. We believe our sophisticated approaches overcame the potential limitations of tumor cell and immune cell evaluation in the TCGACOADREAD cohort. Considering that TMB is widely accepted as a marker of an ICI treatment response, ${ }^{31} 32$ our results suggest that an indel mutation load may be a useful biomarker for precise patient stratification of the ICI treatment response in patients with MSI-high tumors.

POLE mutations are infrequently encountered in clinical sequencing studies of solid malignant tumors, but they have attracted the attention of clinicians because POLE inactivating mutations are believed to be associated with a very high TMB, an increased tumor immune response and a better response to ICIs. ${ }^{33}$ Nevertheless, it has been also widely recognized that not all POLE gene mutations affect the biologic activity of the protein product of the $P O L E$ gene. ${ }^{34}$ We also found an ultrahypermutated group with ONC/LO POLE mutations in the CRC cases, while the unknown-significant and truncated POLE-mutated 
group may not show hypermutation. Interestingly, we detected two cases with concurrent MSI-high and multiple unknown significance POLE mutations showing ultrahypermutation genotype. Recently, Haradhvala and colleagues reported that polymerase proofreading deficiency precedes the MMR deficiency in concurrent polymerase proofreading and MMR deficient cases in TCGA endometrial cancer cohort. ${ }^{35}$ On the contrary, we observed that MSH6 and MSH2 mutations preceded the multiple POLE mutations in our first concurrent POLEmutated and MMR-deficient colon cancer case, while we could not determine the two mutation events temporally in the second case. It should be noted that these two tumors showed distinct mutational characteristics (ultrahypermutation, high numbers of single nucleotide variants and a low indel mutation load), which is more like the genotype of POLE-inactivated tumors rather than MSI-high tumors. Even though it is virtually impossible to conclude whether the two genomic events (POLE or MMR gene mutation) occurred simultaneously or were temporally separated in our cases, we strongly believe that the unknown-significant POLE mutations could be a secondary mutation to MMR deficiency that have an impact on POLE function, resulting in an increased TMB in concurrent POLE-mutated and MMR-deficient tumors.

A number of studies have suggested that the ultrahypermutation caused by POLE inactivation is associated with an increased immune reaction against the tumor and a better prognosis in endometrial cancer ${ }^{36} 37$ and CRCs. ${ }^{38}$ Also, there are several reports showing clinical responses to ICI treatment in POLE-inactivated malignancies. ${ }^{39-41}$ The most interesting point in this regard is that tumor immune responses of POLE-inactivated tumors appear to be heterogeneous or even contradictory. Shia et alreported that more than half of POLE-mutated CRCs showed histologic features just like those of conventional CRCs. ${ }^{42}$ In addition, Wang et al demonstrated that not all POLEinactivated CRCs displayed high CD8 + T cell infiltration and a clinical response to ICI treatment. ${ }^{43}$ Concordantly, we demonstrated that the tumors harboring unknownsignificant POLE mutations are not usually associated with ultrahypermutation, and even in the ultrahypermutated CRC cases harboring ONC/LO POLE mutation, tumorous immune signatures or increased immune cell densities were not found to be significantly increased in both our AMC and TCGA-COADREAD cohorts. Therefore, we concluded that the tumorous immunogenic potential of novel POLE mutations, whether or not the mutations are within the exonuclease domain as well as single or multiple mutations, should be interpreted in the context of their mutational profile and tumorous immune cell infiltration because an ultrahypermutation may not always lead to an increased tumor immune reaction or a favorable ICI treatment response.

Of note, we found that the genes involved in the PI3K/ AKT $/ \mathrm{mTOR}$ pathway were frequently altered in the MSIhigh group. Several studies using small subsets of MMRdeficient CRCs revealed that PIK3CA gene mutations were more frequently identified in MMR-deficient than MMR-proficient CRCs with frequencies ranged from $30 \%$ to $40 \% .{ }^{44}{ }^{45}$ Furthermore, PTEN promoter inactivation and/or sporadic mutations are also common in MMRdeficient CRCs. ${ }^{46}$ In this study, the frequency of PI3K/ AKT/mTOR pathway mutations $(75 \%)$ in MSI-high tumors was higher than in previous reports, probably because we examined many PI3K pathway-related genes, including PIK3R1, PIK3R2 and AKT1. Recent studies implicated PI3K/AKT/mTOR pathway activation in the escape of immune surveillance of tumors by regulating chemokine and/or immune checkpoint signaling, ${ }^{48}$ which also suggests the role of this pathway in immune evasion and cancer progression. These findings suggest that mutational activation of the $\mathrm{PI} 3 \mathrm{~K} / \mathrm{AKT} / \mathrm{mTOR}$ pathway may exert an important role in the pathogenesis of MSI-high malignancies and targeting the PI3K/AKT/ mTOR pathway in combination with ICI treatment could be an efficient treatment strategy, which has been demonstrated in several pre-clinical models. ${ }^{49} 50$

In this study, we showed that indel mutation burden rather than the total TMB could be a predictor of a TIL density in MSI-high and POLE-mutated tumors. We also found that not all ultrahypermutated ONC/LO POLEmutant tumors are directly associated with increased immune cell infiltration, suggesting the immunogenic heterogeneity of POLE-inactivated malignancies. MSIhigh tumors with multiple uncharacterized POLE mutations may have similar genomic characteristics as $\mathrm{ONC} /$ LO POLE-mutated tumors, which suggests that MMR deficiency may take precedence over the POLE mutations, which may be pathogenic.

Contributors Study conceptualization and design: JC. Data collection: DK. Analysis and interpretation of results: HSH, DK and JC. Draft manuscript preparation: HSH. All authors reviewed the results and approved the final version of the manuscript.

Funding This work was supported by the National Research Foundation of Korea (NRF) grant (No. 2020R1A2C2006815) funded by the Korean government (MSIT).

Competing interests None declared.

Patient consent for publication Not required.

Ethics approval Institutional Review Board of Asan Medical Center (Approval No. 2018-1080).

Provenance and peer review Not commissioned; externally peer reviewed.

Data availability statement No data are available. Sharing of sequencing data has been rejected upon the request of Institutional Review Board.

Supplemental material This content has been supplied by the author(s). It has not been vetted by BMJ Publishing Group Limited (BMJ) and may not have been peer-reviewed. Any opinions or recommendations discussed are solely those of the author(s) and are not endorsed by BMJ. BMJ disclaims all liability and responsibility arising from any reliance placed on the content. Where the content includes any translated material, BMJ does not warrant the accuracy and reliability of the translations (including but not limited to local regulations, clinical guidelines, terminology, drug names and drug dosages), and is not responsible for any error and/or omissions arising from translation and adaptation or otherwise.

Open access This is an open access article distributed in accordance with the Creative Commons Attribution Non Commercial (CC BY-NC 4.0) license, which permits others to distribute, remix, adapt, build upon this work non-commercially, and license their derivative works on different terms, provided the original work is properly cited, appropriate credit is given, any changes made indicated, and the use is non-commercial. See http://creativecommons.org/licenses/by-nc/4.0/. 
ORCID iD

Hee Sang Hwang http://orcid.org/0000-0001-9486-9214

\section{REFERENCES}

1 Vinay DS, Ryan EP, Pawelec G, et al. Immune evasion in cancer: mechanistic basis and therapeutic strategies. Semin Cancer Biol 2015;35 Suppl:S185-98.

2 Chan TA, Yarchoan M, Jaffee E, et al. Development of tumor mutation burden as an immunotherapy biomarker: utility for the oncology clinic. Ann Oncol 2019;30:44-56.

3 Marcus L, Lemery SJ, Keegan P, et al. Fda approval summary: pembrolizumab for the treatment of microsatellite Instability-High solid tumors. Clin Cancer Res 2019;25:3753-8.

4 Mehnert JM, Panda A, Zhong $\mathrm{H}$, et al. Immune activation and response to pembrolizumab in POLE-mutant endometrial cancer. $J$ Clin Invest 2016;126:2334-40.

5 Wang F, Zhao Q, Wang Y-N, et al. Evaluation of POLE and POLD1 mutations as biomarkers for immunotherapy outcomes across multiple cancer types. JAMA Oncol 2019;5:1504-6.

6 Maleki Vareki S. High and low mutational burden tumors versus immunologically hot and cold tumors and response to immune checkpoint inhibitors. J Immunother Cancer 2018;6:157.

7 McGranahan N, Furness AJS, Rosenthal R, et al. Clonal neoantigens elicit $\mathrm{T}$ cell immunoreactivity and sensitivity to immune checkpoint blockade. Science 2016;351:1463-9.

8 Fridman $\mathrm{WH}$, Pagès $\mathrm{F}$, Sautès-Fridman $\mathrm{C}$, et al. The immune contexture in human tumours: impact on clinical outcome. Nat Rev Cancer 2012;12:298-306.

9 Masucci GV, Cesano A, Hawtin R, et al. Validation of biomarkers to predict response to immunotherapy in cancer: Volume I - preanalytical and analytical validation. J Immunother Cancer 2016;4:76.

10 Sade-Feldman M, Yizhak K, Bjorgaard SL, et al. Defining T cell states associated with response to checkpoint immunotherapy in melanoma. Cell 2018;175:998-1013.

11 Wu S-P, Liao R-Q, Tu H-Y, et al. Stromal PD-L1-Positive Regulatory $T$ cells and PD-1-Positive CD8-Positive T cells Define the Response of Different Subsets of Non-Small Cell Lung Cancer to PD-1/PD-L1 Blockade Immunotherapy. J Thorac Oncol 2018;13:521-32.

12 Cristescu R, Mogg R, Ayers M, et al. Pan-tumor genomic biomarkers for PD-1 checkpoint blockade-based immunotherapy. Science 2018;362:eaar3593.

13 Li H, Durbin R. Fast and accurate short read alignment with BurrowsWheeler transform. Bioinformatics 2009;25:1754-60.

14 McKenna A, Hanna M, Banks E, et al. The genome analysis toolkit: a MapReduce framework for analyzing next-generation DNA sequencing data. Genome Res 2010;20:1297-303.

15 Lai Z, Markovets A, Ahdesmaki M, et al. VarDict: a novel and versatile variant caller for next-generation sequencing in cancer research. Nucleic Acids Res 2016;44:e108.

16 Talevich E, Shain AH, Botton T, et al. CNVkit: genome-wide copy number detection and visualization from targeted DNA sequencing. PLoS Comput Biol 2016;12:e1004873.

17 Robinson JT, Thorvaldsdóttir H, Winckler W, et al. Integrative genomics viewer. Nat Biotechnol 2011;29:24-6.

18 Chakravarty D, Gao J, Phillips SM. OncoKB: a precision oncology knowledge base. JCO Precis Oncol 2017;2017:PO.17.00011.

$19 \mathrm{Kim}$ JE, Chun S-M, Hong YS, et al. Mutation burden and I index for detection of microsatellite instability in colorectal cancer by targeted next-generation sequencing. J Mol Diagn 2019;21:241-50.

20 Umar A, Boland CR, Terdiman JP, et al. Revised Bethesda guidelines for hereditary nonpolyposis colorectal cancer (Lynch syndrome) and microsatellite instability. J Natl Cancer Inst 2004;96:261-8.

21 Mayakonda A, Lin D-C, Assenov Y, et al. Maftools: efficient and comprehensive analysis of somatic variants in cancer. Genome Res 2018;28:1747-56.

22 Bankhead P, Loughrey MB, Fernández JA, et al. QuPath: open source software for digital pathology image analysis. Sci Rep 2017;7:16878.

23 Cerami E, Gao J, Dogrusoz U, et al. The cBio cancer genomics portal: an open platform for exploring multidimensional cancer genomics data. Cancer Discov 2012;2:401-4.

24 Gao J, Aksoy BA, Dogrusoz U, et al. Integrative analysis of complex cancer genomics and clinical profiles using the cBioPortal. Sci Signal 2013;6:pl1.

25 Thorsson V, Gibbs DL, Brown SD, et al. The immune landscape of cancer. Immunity 2018;48:812-30.

26 Boyiadzis MM, Kirkwood JM, Marshall JL, et al. Significance and implications of FDA approval of pembrolizumab for biomarkerdefined disease. J Immunother Cancer 2018;6:35.
27 Ishikawa T, Fujita T, Suzuki Y, et al. Tumor-Specific immunological recognition of frameshift-mutated peptides in colon cancer with microsatellite instability. Cancer Res 2003;63:5564-72.

28 Saeterdal I, Bjørheim J, Lislerud K, et al. Frameshift-mutationderived peptides as tumor-specific antigens in inherited and spontaneous colorectal cancer. Proc Natl Acad Sci U S A 2001;98:13255-60.

29 Maby P, Tougeron D, Hamieh M, et al. Correlation between density of CD8+ T-cell infiltrate in microsatellite unstable colorectal cancers and frameshift mutations: a rationale for personalized immunotherapy. Cancer Res 2015;75:3446-55

30 Tougeron D, Fauquembergue E, Rouquette A, et al. Tumor-Infiltrating lymphocytes in colorectal cancers with microsatellite instability are correlated with the number and spectrum of frameshift mutations. Mod Pathol 2009;22:1186-95.

31 Loupakis F, Depetris I, Biason P, et al. Prediction of benefit from checkpoint inhibitors in mismatch repair deficient metastatic colorectal cancer: role of tumor infiltrating lymphocytes. Oncologist 2020;25:481-7.

32 Schrock AB, Ouyang C, Sandhu J, et al. Tumor mutational burden is predictive of response to immune checkpoint inhibitors in MSI-high metastatic colorectal cancer. Ann Oncol 2019;30:1096-103.

33 Nebot-Bral L, Brandao D, Verlingue L, et al. Hypermutated tumours in the era of immunotherapy: the paradigm of personalised medicine. Eur J Cancer 2017;84:290-303.

34 Campbell BB, Light N, Fabrizio D, et al. Comprehensive analysis of hypermutation in human cancer. Cell 2017;171:1042-56.

35 Haradhvala NJ, Kim J, Maruvka YE, et al. Distinct mutational signatures characterize concurrent loss of polymerase proofreading and mismatch repair. Nat Commun 2018;9:9.

36 Bellone S, Centritto F, Black J, et al. Polymerase $\varepsilon$ (pole) ultramutated tumors induce robust tumor-specific CD4+ T cell responses in endometrial cancer patients. Gynecol Oncol 2015;138:11-17.

37 van Gool IC, Eggink FA, Freeman-Mills L, et al. Pole proofreading mutations elicit an antitumor immune response in endometrial cancer. Clin Cancer Res 2015;21:3347-55

38 Domingo E, Freeman-Mills L, Rayner E, et al. Somatic pole proofreading domain mutation, immune response, and prognosis in colorectal cancer: a retrospective, pooled biomarker study. Lancet Gastroenterol Hepatol 2016;1:207-16.

39 Gong J, Wang C, Lee PP, et al. Response to PD-1 Blockade in Microsatellite Stable Metastatic Colorectal Cancer Harboring a POLE Mutation. J Natl Compr Canc Netw 2017;15:142-7.

40 Lee L, Ali S, Genega E. Aggressive-Variant microsatellite-stable pole mutant prostate cancer with high mutation burden and durable response to immune checkpoint inhibitor therapy. JCO Precis Oncol 2018;2.

41 Silberman R, Steiner DF, AA L. Complete and prolonged response to immune checkpoint blockade in POLE-Mutated colorectal cancer. JCO Precis Oncol 2019;3:1-5.

42 Shia J, Schultz N, Kuk D, et al. Morphological characterization of colorectal cancers in the cancer genome atlas reveals distinct morphology-molecular associations: clinical and biological implications. Mod Pathol 2017;30:599-609.

43 Wang C, Gong J, Tu TY, et al. Immune profiling of microsatellite instability-high and polymerase $\varepsilon(P O L E)$-mutated metastatic colorectal tumors identifies predictors of response to anti-PD-1 therapy. J Gastrointest Oncol 2018;9:404-15.

44 Cohen SA, Turner EH, Beightol MB, et al. Frequent PIK3CA mutations in colorectal and endometrial tumors with 2 or more somatic mutations in mismatch repair genes. Gastroenterology 2016;151:440-7.

45 Li W, Qiu T, Dong L, et al. Prevalence and characteristics of PIK3CA mutation in mismatch repair-deficient colorectal cancer. J Cancer 2020;11:3827-33.

46 Goel A, Arnold CN, Niedzwiecki D, et al. Frequent inactivation of PTEN by promoter hypermethylation in microsatellite instability-high sporadic colorectal cancers. Cancer Res 2004;64:3014-21.

47 Lin El, Tseng L-H, Gocke CD, et al. Mutational profiling of colorectal cancers with microsatellite instability. Oncotarget 2015;6:42334-44.

48 Xue G, Zippelius A, Wicki A, et al. Integrated Akt/PKB signaling in immunomodulation and its potential role in cancer immunotherapy. $J$ Natl Cancer Inst 2015;107:djv171.

49 Borcoman E, De La Rochere P, Richer W, et al. Inhibition of PI3K pathway increases immune infiltrate in muscle-invasive bladder cancer. Oncoimmunology 2019;8:e1581556.

50 Carnevalli LS, Sinclair C, Taylor MA, et al. PI3Ka/ठ inhibition promotes anti-tumor immunity through direct enhancement of effector CD8 ${ }^{+}$T-cell activity. J Immunother Cancer 2018;6:158. 